VOX PATRUM $22(2002)$ t. 42.43

Adam NYK SDB

(Kraków)

\title{
IMMAGINE EVANGELICA DI MARIA MADRE DI GESÙ NEI PRIMI TRE SECOLI
}

Maria madre di Gesù Cristo di Nazareth viene venerata sopratutto in

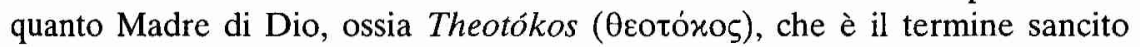
dal Concilio d'Efeso nel 431. Alcuni teologi sostengono che il questo titolo sarebbe stato usato nei confronti della madre di Gesù già nel corso dei primi secoli. Vogliamo vedere se, effettivamente, esso sia stato mai usato in questo periodo in senso cristiano esponendo i dati discussi dai vari autori.

Prima di iniziare il nostro lavoro ci soffermiamo sul termine Theotókos, presentandolo anzitutto dal punto di vista grammaticale e sucessivamente cercando di riportandolo alla problematica riguardante gli inizi dell'uso cristiano di questo termine, cercando di provare se esso sia mai stato usato nei tre primi secoli. Partiamo con una breve esposizione dei dati biblici. Analizziamo poi l'accoglienza del messaggio evangelico da parte degli autori dell'età subapostolica, nel II e nel III secolo, evidenziando il caso di Origene; concludiamo con la valutazione dei testi dalla seconda parte del secolo III fino agli inizi del IV.

1. Analisi grammaticale del termine Theotókos. Il sostantivo femminile

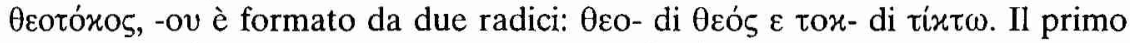
elemento: $\theta \varepsilon o ́ \zeta$, -oṽ è comunemente usato nella lingua greca per indicare il dio, la dea, la divinità. La parola deriva dalla radice indoeuropea $<^{*}$ dhēs- $/<^{*}$ dhas- e $<$ *dheues- con i significati di: mettere, tempio, festa, spirito ${ }^{1}$. In greco il sostantivo, ,indica sia la divinità femminile che maschile"2, anche se spesso per una dea viene usato $\theta \varepsilon \alpha$, che è un derivato. A volte il vocabolo viene adoperato per semidei o per eroi o per persone altamente ragguardevoli e venerande ${ }^{3}$. La seconda componente è quella del verbo $\tau i x \tau \omega, \tau l-\tau x \omega$, raddoppiamento arcaico

${ }^{1}$ Cf. J. Pokorny, Indogermanisches etymologisches Wörterbuch, Bern - München 1959, 259; cf. anche C.D. Buck, A Dictionary of selected synonyms in the principal Indo-European Languages, Chicago - London 1949, 1464. Questa radice è diversa dalla: <" dèiuo-s / <" deiüi-, con i significati di: brillare, luce del giorno, usata in greco in forma dios per indicare la divinità, cf. J. Pokorny, op. cit., p. 185.

${ }^{2}$ F. Montanari, Vocabolario della lingua greca, Torino 1995, 907.

${ }^{3}$ Cf. B. Bonazzi, Dizionario greco-italiano, Napoli 1931, 504. 
del presente al grado debole; l'indoeuropeo <*tek- significa: mettere al mondo, generare, partorire, fruttificare ${ }^{4}$. Il verbo viene riferito sia agli dei che agli uomini, ma anche agli animali o alla produzione della terra ${ }^{5}$. A differenza

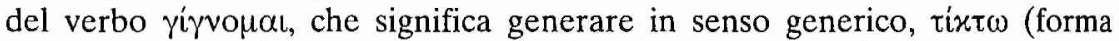
attiva e transitiva) di solito viene riferito all'attività genitrice propria di una madre (= partorire). Da questo verbo derivano le forme sostantivate, parallele

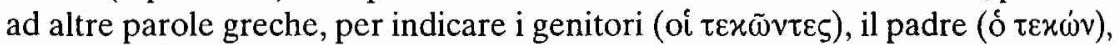

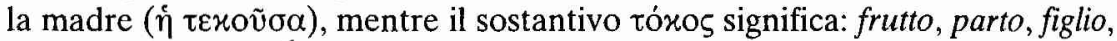
interesse, guadagno ${ }^{6}$.

Da queste due radici risulta il composto $\theta \varepsilon o \tau o ́ x o 5$, aggettivo con valore sostantivato, in quanto i due concetti, l'uno principale di agente, l'altro secondario di atto specificante l'azione, „costituiscono un predicato unico, a sé stante, che qualifica e simultaneamente specifica e determina inequivocabilmente la persona, a cui è riferito"7 . Così dice Roberto Iacoangeli, aggiungendo che „il vocabolo fa parte di quei gruppi di composti che sono definiti esocentrici, nei quali l'accento cade su quello dei due componenti, che regge sintatticamente l'altro, svolgendo il ruolo di soggetto agente. Così $\mathfrak{\eta} \theta \varepsilon$ cotóxo s significa Colei che ha partorito Dio; ed è, pertanto, la Genitrice di Dio, la Madre di Dio"8. Infatti - prosegue il nostro autore - ,la radice to $x$, che costituisce il secondo dei due componenti di $\theta$ cotóxos, è radice forte-perfettiva, da cui prende forma

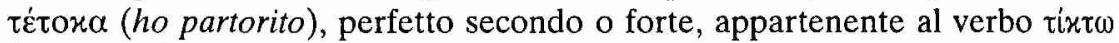
(partorisco). Ora, la forma perfettiva indica, appunto, un'azione realmente

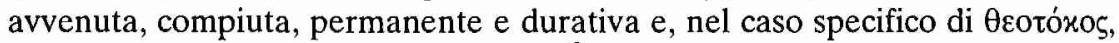
ovviamente irreversibile e immutabile" ${ }^{9}$. Questo „conio riflette altri termini di simile stampo di provenienza classica, come ad esempio $\theta$ coфógos (Deum

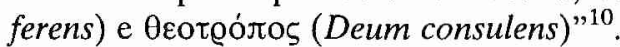

Concludendo quest'analisi notiamo che in età bizantina da questo termine sono stati costruiti i derivati, come ad esempio $\theta$ cotóxoร, -on (l'accento sulla prima „o") con il significato di: partorito da dio, o nato da Dio ${ }^{11}$, vale a dire: figliolfiglia di dio ${ }^{12}$, oppure $\theta$ cotoxiov: un inno rivolto a Maria Vergine, o la conclusione mariana di un tropario o di una ode ${ }^{13}$.

${ }^{4}$ Cf. J. Pokorny, op. cit. p. 1057.

${ }^{5}$ Cf. F. Montanari, op. cit., p. 2003.

${ }^{6}$ Cf. ibidem, p. 2015

${ }^{7}$ R. lacoangeli, ,Sub tuum prosidium”. La più antica preghiera mariana: filologia e fede, in: S. Felici (ed.), La mariologia nella catechesi dei Padri (età prenicena), Roma 1989, 214-215.

8 Ibidem, p. 215.

9 Ibidem.

10 Ibidem.

${ }^{11}$ Cf. H. Stephanus, Thesaurus Graecae linguae, V, Graz 1954, 313, dove @eotóxoร viene tradotto: $A$ Deo natus.

${ }^{12}$ Cf. J. Planche, Dictionnaire Grec-Français, composé sur le „Thesaurus linguae Graecae" de Henri Estienne, Paris 1845. A pagina 603 si dice che questo termine indica „fils ou fille d'un dieu”. 
Circa la paternità del titolo 0عotóxos le opinioni degli studiosi sono contrastanti. Vengono proposti diversi autori che avrebbero usato per la prima volta questo termine ${ }^{14}$.

2. Maria nel Nuovo Testamento. I testi del Nuovo Testamento presentano Maria di Nazaret come la madre di Gesù Cristo, anche se non si usa mai il termine Theotókos. Questi testi sono stati successivamente ripresi dalla teologia come punti di partenza, per sviluppare il concetto della maternità divina di Maria. Facciamo una breve analisi di questi passi, seguendo l'ordine cronologico della redazione dei testi, notando che le testimonianze del Nuovo Testamento riguardanti il ruolo materno di Maria si possono suddividere in tre tappe ${ }^{15}$.

La prima, che è la più antica, è rappresentata dalla Lettera di san Paolo ai Galati, scritta probabilmente negli anni cinquanta d.C., e dal Vangelo di Marco, composto prima del $70 \mathrm{~d}$.C. La seconda tappa è costituita dai Vangeli di Matteo e di Luca, redatti dopo il 70, mentre la terza riguarda il Vangelo di Giovanni e l'Apocalisse, che ci portano tra la fine del I e l'inizio e del II secolo cristiano.

Dal punto di vista cronologico il primo testo relativo a Maria è Gal 4,4: „Quando venne la pienezza del tempo, Dio mandò suo figlio, nato da donna $\left(\gamma u v \eta^{\prime}\right) " 16$. Paolo mette in luce la prospettiva storico-salvifica in cui Dio, nella pienezza escatologica dei tempi, manda il suo Figlio nato da donna. Così questa donna viene collocata nel posto più vicino al compimento escatologico, come la creatura più prossima al cuore del mistero, al „centro escatologico” della storia. Il passo di san Paolo, che per la prima volta mette in luce la maternità di Maria, rispecchia la più antica intuizione del significato storico-salvifico di Maria: il suo ruolo consiste nel fatto di essere la madre del Figlio di Dio.

Il Vangelo di Marco è molto sobrio nel trattare il rapporto di Gesù con sua madre. „I due soli testi di Marco sulla „Madre di Gesù” (Mc 3, 31-35 e 6, 1-6)

${ }^{13}$ A Patristic Greek Lexicon spiega così il termine; „hymn addressed to BMV; of concluding troparion or an ode" (G.W.H. Lampe, A Patristic Greek Lexicon, Oxford 1961, 641). Un altro derivato riportato da questo Lexicon è @eotóxvos, tradotto „born of God".

${ }^{14}$ A questo proposito A.M. Triacca mette in evidenza alcune incongruenze presenti nel Nuovo Dizionario di Mariologia (= S. De Fiores [ed.], Nuovo Dizionario di Mariologia, Cinisello Balsamo 1985), dove si legge ,a p. 1388 che è Origene il primo testimone conosciuto della denominazione $(=\mathrm{T}$. Koehler). Mentre a p. 1142 si legge che il titolo Theotókos sarebbe il termine tecnico usato per la prima volta da Atanasio nel 373 in trattazioni teologiche ( $=\mathrm{E}$. Lodi). Infine a p. 1056 si legge che bisogna ricordare i frammenti di Alessandro di Alessandria $(\dagger 325)$ dove per la prima volta ricorre il titolo Theotókos (= E. Toniolo)"; cf. A.M. Triacca, „Sub tuum prasidium": nella „lex orandi" un'anticipata presenza della ,lex credendi". La „teotocologia” precede la "mariologia"?, in: S. Felici (ed.), op. cit., p. 199, nota 71.

is Seguiamo l'impostazione di B. Forte, Maria, la donna icona del Mistero, Milano 1989, 45-103.

${ }^{16}$ Tutte le citazioni greche del Nuovo Testamento sono prese dall'edizione E. e E. Nestle B. e K. Aland, The Greek New Testament, Stuttgart $1993^{27}$. 
hanno [...] un carattere occasionale. Hanno, per di più, un carattere negativo" ${ }^{\prime 17}$. L'Evangelista, infatti, presenta il distacco di Gesù dai legami di parentela e di sangue: egli preferisce la parentela di fede di chi compie la volontà di Dio. Non si dice che la madre faccia parte della sua famiglia spirituale o che lo comprenda e lo onori come un profeta. Si intravede che il ruolo principale di Maria consiste nella sua maternità: infatti Gesù viene chiamato "figlio di Maria", che è un'espressione insolita, perché un figlio era sempre chiamato con il nome di suo padre.

Nel secondo periodo i Vangeli di Matteo e di Luca seguono un certo approfondimento pasquale dell'evento di Cristo. Matteo cerca di instaurare un parallelismo tra la storia di Gesù e i racconti dell'Antico Testamento. Così la nascita di Gesù è una seconda creazione: come lo Spirito aleggiava sulle acque primordiali, così ora opera in Maria che lo accoglie (cf. Mt 1, 18-20 con Gen 1,2). Maria è il grembo della nuova creazione, dove si compie l'incarnazione e la nascita verginale del Figlio di Dio. Luca presenta in modo positivo il rapporto tra la famiglia fisica e quella escatologica di Gesù. La scena dell'annunciazione (Lc 1, 26-38) richiama il concetto di alleanza, dove si ha un mediatore che annuncia la volontà di Dio e il popolo che risponde. Abbiamo l'angelo Gabriele come intermediario e Maria che rappresenta l'Israele Santo; ella, dando la sua risposta di fede, permette a Dio di stabilire la nuova e perfetta alleanza. da sottolineare la prontezza e la sottomissione di Maria nella sua risposta positiva: Fiat (in greco il verbo è all'ottativo: $\gamma \varepsilon$ voıro, „che avvenga"), che esprime il desiderio di collaborare con Dio. Luca vede l'azione dello Spirito in Maria come una svolta nella storia della salvezza, mostrando l'esperienza di grazia vissuta da Maria per l'azione di Dio che la rende madre del

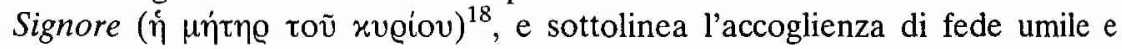
disponibile con cui ella ha risposto. Questa fede, poi, ha un itinerario progressivo, è un cammino di colei che ,custodiva tutte le cose, meditandole nel suo cuore" (Lc 2, 19. 51), e per questo Maria diventa il modello del discepolo, che comprende sempre più il disegno di salvezza come il mistero della croce. Maria è presentata come ,associata in modo assolutamente unico alla vita di Gesù. Presente in importanti momenti della sua vita privata e pubblica, ella si trova in mezzo agli apostoli al momento della discesa dello Spirito Santo" ${ }^{19}$, ed è perciò all'interno della prima comunità ecclesiale.

Il terzo momento è costituito dall'opera giovannea, redatta tra la fine del I e l'inizio del II secolo d.C. Il Vangelo di Giovanni presenta Maria all'inizio e alla conclusione dei segni compiuti da Gesù. A Cana (Gv 2,1-12) la madre di Cristo

17 R. Laurentin, Compendio di mariologia, Roma 1957, 21.

18 Cf. Lc $1,43$.

${ }^{19}$ R.C . Turner, Maria, in: N. Lossky - J.M. Bonino ed altri, Dizionario del movimento ecumenico, Bologna 1994, 700. 
collabora al primo miracolo compiuto dal suo Figlio. L'importanza di questo gesto deriva dal fatto che nella teologia simbolica giovannea questo miracolo è l"'archetipo" di tutti gli altri. Il posto di Maria è particolare: Ella si dimostra attenta alle preoccupazioni degli uomini, chiede al Figlio l'aiuto a loro favore, richiede l'obbedienza a Gesù; insomma fa da intermediaria tra Lui e il popolo. Il segno di Cana anticipa in un certo senso il mistero pasquale come evento di alleanza nuziale, che è un momento decisivo nella storia della salvezza, dove Maria ha un ruolo ben evidenziato dall'Evangelista.

La scena presso la croce ha un denso valore simbolico: qui si compie l'ultimo segno di Gesù e anche qui è presente Maria, la madre di Cristo, che diventa la madre del discepolo fedele. In questo momento Maria viene vista come una madre che assiste soffrendo alla morte redentrice del Figlio. Si discute se, e in che senso, le sofferenze di Maria abbiano un valore salvifico. L'Apocalisse, al capitolo 12, presenta il segno della „donna” che rappresenta la Chiesa, ma ha anche aspetti mariologici. La "donna" viene descritta come madre del Messiare; ora, sicome la persona concreta della madre di Cristo era ben conosciuta,

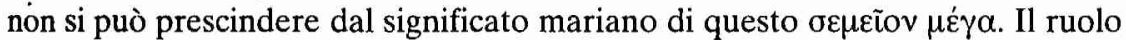
di Maria viene unito perfettamente alle vicende della Chiesa.

Come si vede, il Nuovo Testamento presenta un certo sviluppo nel raccontare il ruolo della Madre di Cristo nella storia della salvezza. In Maria la Scrittura presenta come Dio opera per l'uomo e come la creatura possa dare a Dio la sua risposta libera. Dall'annunciazione Maria ha cooperato, col suo consenso, all'incarnazione redentrice. Con lei Dio ha stretto un'alleanza per il compimento di questo mistero. La partecipazione di Maria all'opera della salvezza si fonda sull'accettazione e il compimento della maternità nei riguardi del Messia, cioè nell'evento dell'incarnazione. La premura materna di Maria prosegue durante tutta la vita di Gesù, fino alla sua morte in croce e alla sua risurrezione e continua nella prima comunità cristiana. L'opera giovannea mette in risalto il valore profondo della presenza di Maria nella comunità ecclesiale: prima come madre del discepolo che rappresenta tutti i credenti, poi come persona connessa strettamente con le vicende della Chiesa ( $A p 12$ ). Comunque dobbiamo riconoscere oggettivamente che Maria nel Nuovo Testamento „occupa un posto materialmente poco importante" ${ }^{20}$. L'importanza del dato biblico sull'impegno di Maria nella salvezza deriva dal fatto che la Scrittura, essendo ispirata, ha valore normativo, e tutta la riflessione teologica che segue deve tenerne conto.

3. Maria vista dagli autori del II secolo. I primi autori cristiani recepiscono tutto il messaggio biblico riguardante Maria vista in chiave cristologica. In

${ }^{20}$ Così R. Laurentin, op. cit., p. 19, anche se, a suo giudizio, questo posto è „profondamente significativo". 
nessun momento si tratta di Maria, quale personaggio isolato. Essa è sempre in funzione di Cristo, come madre del Salvatore, è piena di grazia e di fede e rende testimonianza all'opera del proprio Figlio.

Il punto di partenza è sempre la cooperazione di Maria all'incarnazione del Verbo di Dio. Da sant'Ignazio Antiocheno $(\dagger 110)^{21}$ in poi, si considera la sua maternità come prova della vera umanità di Gesù, contro le eresie e le deviazioni della dottrina cristologica dei primi secoli. Quindi, si comincia a valorizzare il significato salvifico di Maria nella storia della salvezza. I primi autori che vedono questo aspetto sono san Giustino $(\dagger 165)^{22}$ nel Dialogo con Trifone e sant'Ireneo $(\dagger 202)$ nell'Adversus hareses ${ }^{23}$.

$\mathrm{Da}$ Giustino parte il celebre confronto antitipico Eva-Maria. Questo „Apologista è il primo autore che, sull'esempio del parallelismo paolino Adam/Cristo, contrappone Eva a Maria (cf. Dialogo con Trifone 100). Egli mette a confronto due momenti generatori della storia, due scene: quella del peccato originale e quella dell'anunciazione, e rileva l'opposto atteggiamento delle due protagoniste, ambedue vergini. Eva disobbediente, genera la morte; Maria, obbediente, genera la vita. Maria è dunque la donna nuova che apre la via della salvezza con la sua fede. Per quella stessa via per cui era stata rovinata, l'umanità viene salvata. La „donna-vergine" (Eva/Maria) è responsabile della storia umana accanto e in subordine all'uomo-capo (Adamo/Cristo)"24.

Questa idea, ripresa e sviluppata da Ireneo e Tertulliano, risulta molto significativa per il modo in cui viene intesa la figura di Maria. Infatti, la scelta di Eva come un suo tipo è dovuta al fatto di essere una madre. Il nome stesso di Eva (Hawwâh) etimologicamente significa: „Madre di tutti i viventi"25. Ora Maria, madre di Cristo, diventa „nuova Eva”, ovvero „nuova Madre di tutti i viventi”.

Ireneo, noto per il suo atteggiamento benevolo verso le donne, ${ }^{26}$ nell'Adversus hareses 3-5, sottolinea la prontezza di Maria a collaborare con Dio attraverso la fede e l'obbedienza. Il suo è un ruolo necessario alla logica del

${ }^{21}$ Per la mariologia di Ignazio cf. F. Bergamelli, Caratteristiche e originalità della confessione di fede mariana di Ignazio di Antiochia, in: S. Felici (ed.), op. cit., p. 65-78.

${ }^{22}$ Per la mariologia di questo autore, con la rispettiva bibliografia, cf. M. Maritano, La Vergine Maria negli scritti di Giustino Martire. Miti pagani e mistero cristiano, in: S. Felici (ed.), op. cit., p. 101-120.

${ }^{23}$ Per alcuni aspetti del pensiero mariano di Ireneo cf. A. Orbe, La "recirculatión" de la Virgen María en san Ireneo (Adv. haer. III 22, 4, 71), in: S. Felici (ed.), op. cit., p. 79-99.

${ }^{24}$ G. Bosio - E. Dal Covolo - M. Maritano, Introduzione ai Padri della Chiesa. Secoli I e II, Torino $1998^{4}, 164$.

${ }^{25}$ Gen 3, 20 dice: „L'uomo chiamò la moglie Eva, perché essa fu la madre di tutti i viventi”. L'autore biblico ricollega il nome di Eva (Hawwâh) alla vita (hayyâh). Per rendere questo gioco di parole, la Bibbia TOB traduce: „Eva - cioè la Vivente”, cf. Bibbia TOB. Edizione integrale, Torino 1992, 50.

${ }^{26}$ Cf. E. Dal Covolo, Chiesa, Società, Politica. Aree di ,laicità" nel cristianesimo delle origini, Roma 1994, 132-134. 
piano divino, dove si continua il parallelismo tra Maria ed Eva: „Maria, vergine obbediente, è diventata per sé e per tutto il genere umano, causa di salvezza [...]. Ed è così che la disobbedienza di Eva è stata riscattata dall'obbedienza di Maria: poiché ciò che la vergine Eva legò con l'incredulità, Maria l'ha sciolto con la fede". Maria viene vista come avvocata di Eva, perché, come ,il genere umano è stato legato alla morte da una vergine, esso è salvato da una vergine"27. Ireneo attribuisce alla fede e all'obbedienza di Maria un valore salvifico parallelo all'obbedienza redentrice di Cristo. Il contributo della „Nuova Eva” è simile all'opera del „nuovo Adamo".

Come si vede, anche se lungo il secondo secolo non viene proposta esplicitamente una dottrina sulla maternità divina, si può concordare con l'opinione di R. Laurentin, secondo cui „i Padri fin dal secondo secolo avevano detto che il Figlio di Maria era Dio"28.

4. Maria trattata nel III secolo. In questo periodo prosegue la discussione con i doceti che consideravano l'umanità di Cristo solo apparente e non vera. I Padri della Chiesa cercano di dimostrare che il Verbo di Dio era realmente uomo, in quanto aveva assunto l'umanità da Maria, così che essa è madre di

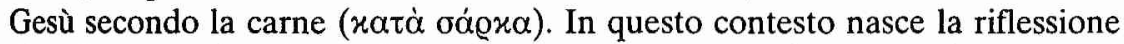
circa la maternità divina di Maria espressa con diversi argomenti e con vari termini linguistici.

Il primo autore cristiano che avrebbe usato il termine Theotókos in riferimento a Maria sarebbe stato Ippolito di Roma ( $\uparrow 235)$. Lo troviamo la prima volta nella edizione greca di una sua opera intitolata Benedizioni di Isaaco e di Giacobbe: „Giuseppe [...] fidanzato a Maria, diventa testimone della madre di Dio"29. La seconda è nella versione siriaca di In Canticum $4,16 .{ }^{30}$ Fa problema però il fatto che tutte e due queste ricorrenze sono considerate inautentiche. Secondo gli studiosi, che hanno edito le opere di Ippolito, la parola Theotókos è un'interpolazione posteriore ${ }^{31}$.

Un altro Padre della Chiesa che tratta l'argomento della maternità di Maria è Clemente Alessandrino ( $† 215 / 216)$. Nei suoi discorsi riguardanti la figura di Cristo, Logos incarnato ${ }^{32}$, afferma: Gesù, „che prese carne e fu concepito nel seno della Vergine, è il Figlio di Dio che ha creato tutto"33, così che Maria è da

${ }^{27}$ Citato da R. Laurentin, Nuova Eva, in: N. Lossky - J.M. Bonino, op. cit., p. 1019-1020.

${ }^{28}$ R. Laurentin, Compendio, op. cit., p. 54, nota 2.

${ }^{29}$ Ibidem.

${ }^{30} \mathrm{Cf}$. ibidem.

${ }^{31}$ Cf. Hippolytus Romanus, Benedictiones Isaac et Iacob, PO 27, 105; cf. idem, In Canticum 4, 16, GCS $1,359$.

${ }^{32}$ Per la dottrina cristologica di Clemente cf. Ch. Kannengiesser, Introduction, in: Athanasius Alexandrinus, De incarnatione, SCh 199, specialmente 69-85.

${ }^{33}$ Stromata VI $15,127, \mathrm{GCS} 52^{4}, 496$. 


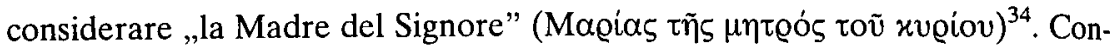
temporaneamente però condanna i circoli teosofici che adorano la madre degli dei: „Maledetto [...] colui che ha introdotto i misteri della madre degli dei”

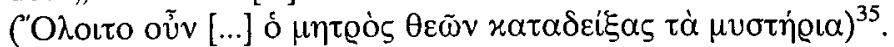

Anche Origene $(\dagger 254)$, il più grande esegeta e teologo di quell'epoca, tratta ampiamente molti argomenti riguardanti la figura della madre di Gesù. Nell'intera sua opera sono numerosi i testi in cui si trovano accenni alla persona di Maria. L'Alessandrino sviluppa diversi aspetti del discorso mariano: la maternità verginale, la santità che la rende tipo del credente spiritualmente perfetto, il ruolo nella redenzione. Vengono confutate le dottrine eterodosse dei doceti, degli ebioniti e degli gnostici. Contro i doceti egli scrisse che Gesù è nato da Maria (ex Maria) e non per Maria (per Mariam). Contro gli ebioniti sottolinea la concezione verginale di Maria. Contro gli gnostici presenta una corretta visione di Gesù (Dio e uomo) e in conseguenza di Maria. L'Alessandrino esegue anche interpretazioni delle parole di Maria trasmesse nei diversi brani evangelici. Da essi vediamo che il ruolo di Maria per lui non si limita „ad essere solo un personaggio fondamentale per una corretta interpretazione del problema cristologico e del discorso ecclesiologico; egli ritiene infatti che tra lei e il credente perfetto vi sia un rapporto di reciproca esemplarità, che lascia trasparire da una parte la dimensione mariana del credente perfetto e dall'altra la perfezione di Maria",36.

I testi origeniani più significativi che si riferiscono più specificamente al tema della maternità di Maria sono le sue catechesi, in particolare le Omelie $X V-X V I I$ sul Vangelo di Luca 2, 25-28 e le Omelie XVIII-XX su Luca 2, 41-52. Secondo lo storico Socrate ( $† 439)$, egli, parlando di Maria nel Commento ai Romani, avrebbe addirittura usato il termine Theotókos mentre „spiega in che

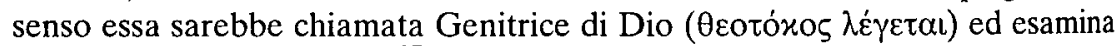
lungamente questo punto"37.

Secondo alcuni studiosi sarebbe stato proprio il maestro alessandrino ad inventare l'appellativo, salvo che la preghiera Sub tuum prasidium non fosse già scritta ancora prima ${ }^{38}$. Questa attribuzione però non può essere accettata pacificamente. Tutte le testimonianze origeniane dell'uso del termine sono

${ }^{34}$ Ibidem I 21, 28, GCS 52. 91.

35 Protrepticus II 13, 3, ed. M. Marcovich, Leiden - New York - Köln 1995, 22.

${ }^{36}$ F. Cocchini, Maria in Origene. Osservazioni storico-dottrinali, in: S. Felici (ed.), op. cit., p. 140.

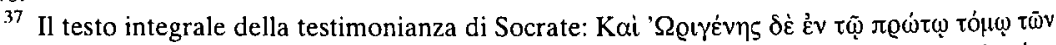

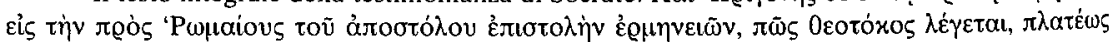
$\grave{\varepsilon} \xi \dot{\eta} \tau \alpha \sigma \varepsilon v$ (HE VII 32, 17, ed. G.Chr. Hansen, GCS Neue Folge 1, Berlin 1995, 381); cf. anche G. Bosio - E. Dal Covolo-M. Maritano, Introduzione ai Padri della Chiesa. Secoli II e III, Torino $1998^{4}$, p. 334, nota 59.

${ }^{38} \mathrm{Cf}$. ibidem. Questo ragionamento è sostanzialmente condiviso da L. Gambero, Culto, in: Nuovo Dizionario di Mariologia, op. cit., p. 429-430. 
dubbie. Per di più, questo titolo non concorda esattamente con la cristologia di Origene $^{39}$. Per l'Alessandrino Gesù Cristo è vero Dio e vero uomo

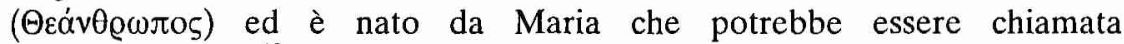
$\Theta \varepsilon a v \theta \varrho \omega \pi \operatorname{có}{ }_{0} \varsigma^{40}$. Per lui Maria è colei che generò il Dio-Uomo ( $\mu$ ía $\dot{\eta}$

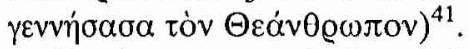

Un altro passo atribuito ad Origene è un frammento della Omelia III, dove

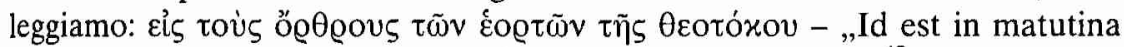
tempora Genitricis Dei, paulum ab initio hæc Origenis laudat"42. Anche questa espressione, come pure i passi precedenti, sono trasmessi solo dalle catene greche e non trovano alcuna conferma nella traduzione latina di Girolamo ${ }^{43}$.

S. Alvarez Campos nel primo volume del suo Corpus Marianum Patristi$\mathrm{Cum}^{44}$ riporta i frammenti origeniani al riguardo della maternità di Maria. Origene nel suo commento al Vangelo di Luca analizza la visita ad Elisabetta, ma non esce fuori dal linguaggio biblico. In Lucam 7 - Maria è Mater Domini ${ }^{45}$; In Lucam 8 - commenta il Magnificat e chiama Maria Mater Salvatoris ${ }^{46}$ e lo ripete più volte, ad es.: In Lucam 9 - Mater Domini et ipsius Mater Salvatoris ${ }^{47} \mathrm{e}$ In Lucam 14 - Mater vero Domini ${ }^{48}$; In Lucam 17 abbiamo Mater Domini ${ }^{49}$.

In nessuno degli altri frammenti greci di Origene, riportati da S. Alvares Campos, si trova l'espressione Theotókos. Origene usa sempre i termini biblici quando si riferisce a Maria. Nei Fragmenta graeca 31 e 32 vengono riportate e

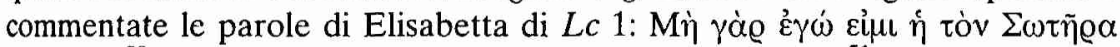

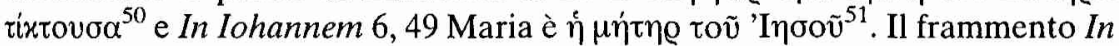

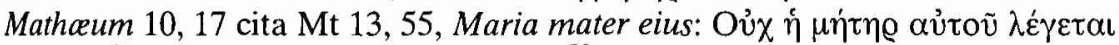
Mapia ${ }^{52}$ e lo ripete In Iohannem 19, $2^{53}$.

${ }^{39}$ Per la cristologia e mariologia di Origene cf. F. Fournier, Introduction, in: Origène, Homélies sur s. Luc, SCh 87bis, 11-92, specialmente 20-22.

${ }^{40}$ Passi riportati dalla edizione delle opere di Origene di J.P. Migne: Fragmenta in Lucam, PG 13, 1901-1910. Queste espressioni non si trovano nella recente edizione critica di M. Rauer, cf. Origenes, In Lucam 7, SCh 87, 154-162 (versione latina di Girolamo) e Fragmenta graca. In Lucam 7,478 e 480.

${ }^{41}$ Cf. Fragmenta in Lucam, PG 13, 1901; cf. nota precedente.

${ }^{42}$ Ibidem, PG 13, 1901, versio latina - 1902.

${ }^{43}$ Cf. In Lucam, PG 13, 1802-1901, in particolare Homilia VII, PG 13, 1817-1819; nuova edizione: SCh 87, 154-226.

${ }^{44}$ Cf. Corpus Marianum Patristicum, I-VIII, Burgos 1970-1985 (= CMP).

45 GCS $49,41-46$, nr 165, CMB I 76

${ }^{46}$ GCS 49, 47-51, nr 170, CMB I 78.

${ }^{47}$ GCS 49, 53, nr 172, CMB I 79.

${ }^{48}$ GCS 49, 85-91, nr 174, CMB I 80.

${ }^{49}$ GCS $49,100-107$, nr 179, CMB I 82.

${ }^{50}$ GCS 49, 239, CMB I 85.

${ }^{51}$ GCS 10, 157, CMB I 88.

52 GCS 40, 21, CMB I 108.

${ }^{53}$ GCS 10, 300, CMB I 125. 
Da tutti i testi origeniani a noi pervenuti, sia nella traduzione di Girolamo come nei frammenti greci, risulta che Origene non va oltre la terminologia del Nuovo Testamento e mantiene l',,immagine evangelica" di Maria ${ }^{54}$. Alla base del suo pensiero su Maria troviamo sempre i passi del Nuovo Testamento. La particolarità, però, è costituita dal fatto che il suo metodo allegorico esercita un notevole influsso nel metodo dell'interpretazione di questi dati biblici „mariologici". Questo gli permette di formulare un discorso mariano più articolato e a vari livelli di lettura.

Altre testimonianze, dove appare la parola qeotkoj, sono senza dubbio interpolazioni post-efesine compiute dai copisti e forse anche dai rappresentanti della scuola origeniana preoccupati di difendere l'ortodossia del loro grande maestro ${ }^{55}$. L'appellativo $\theta \varepsilon o$ cóxos ricorre poi anche in altri passi In Lucam e nel Selecta in Deuteronomium, ma sono tutti frammenti spuri, così che non abbiamo alcuna certezza se effettivamente Origene abbia usato questo termine $^{56}$. Post-efesina è anche l'affermazione di Socrate lo Scolastico, il quale, con molta probabilità, commette un anacronismo. In questa situazione possiamo essere solo certi che l'Alessandrino ha affrontato la questione della maternità di Maria, ma a rigore dobbiamo solo costatare che non è possibile provare con sicurezza che Origene conosca ed usi la parola Theotókos.

Nella seconda metà del III secolo abbiamo solo un'unica testimonianza circa l'uso di Theotókos, anch'essa di dubbia validità. La troviamo nella Storia Cristiana di Filippo di Side, il quale dice che Pierio Alessandrino († 290) avrebbe pronunciato un discorso ,intorno alla Genitrice di Dio" ( $\pi \varepsilon \rho i ~ \tau \tilde{\zeta}$ $\Theta \varepsilon o$ tóxov $)^{57}$. Come nei casi precedenti, anche qui vengono sollevati seri dubbi circa la veracità di questa testimonianza.

L'uso della parola Theotókos è attribuito al vescovo di Alessandria Pietro Alessandrino ( $† 311)$, il quale avrebbe scritto: „Il nostro Signore e Dio Gesù Cristo, alla fine dei tempi si è fatto carne, dalla santa e gloriosa Signora nostra

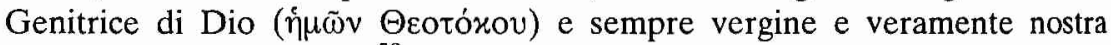

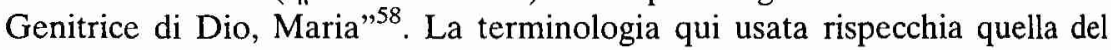
Concilio di Calcedonia (451), e ciò tradisce un'interpolazione fatta almeno un secolo e mezzo più tardi. Anche le altre ricorrenze di questo titolo, attri-

${ }^{54}$ Cf. B. Amata, L'"immagine evangelica" di Maria nei primi Padri (sec. I-V), in: E.M. Toniolo (ed.), La vergine madre nella Chiesa delle origini. Itinerari mariani dei due millenni, I, Roma 1996, 16-23.

55 Come è noto, gli origenisti si sono trovati costretti a giustificare le loro idee più volte, finché non vennero espulsi definitivamente dalla Chiesa dal Concilio di Constantinopoli nel $553 \mathrm{~d}$. C.

${ }^{56}$ Cf. l'affermazione di C. Vagaggini, Maria nelle opere di Origene, Roma 1942, 107: „Dagli scritti di Origene che ora possediamo non si può trarre nessuna certa lezione della parola Theotókos".

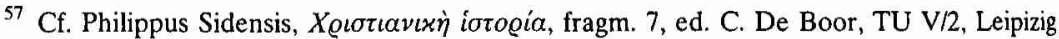
$1888,171,2$.

58 Fragmenta 7, PG 18, 517. 
buite all'imperatore Costantino $(\uparrow 339)^{59}$, a Giovanni Crisostomo $(\dagger 407)$, come pure a molti altri ${ }^{60}$, sono da considerare anacronistiche.

Alla fine di quest'analisi osserviamo che, sicuramente il tema della maternità di Maria è stato trattato nei primi tre secoli ma, non ci sono prove valide che possano rassicurarci che entro la fine del terzo secolo, e forse neppure agli inizi del quarto, il titolo Theotókos sia stato effettivamente usato. Tutti i passi in cui esso ricorre nei testi degli autori di quell'epoca sono da considerare interpolati e spuri. Gli storici, eseguendo la critica dei testi, confermano la facilità con la quale si introduceva nelle opere più antiche il titolo divenuto poi popolare di Theotókos. Questo numero considerevole di testi, con le aggiunte fatte in seguito dalle discussioni cristologiche nei secoli successivi, va spiegato con ,il fervore dei copisti e l'ardore dei polemisti". Così che rimane valida la considerazione di R. Laurentin, secondo cui ,i numerosi testimoni del titolo Theotókos invocati prima del secondo quarto del IV secolo sono tutti inautentici o sospetti"61.

Possiamo quindi concludere che molto verosimilmente nell'arco degli anni del Concilio di Nicea, o almeno fino all'anno 300 dopo Cristo circa, gli autori non vanno oltre il linguaggio espressivo del Nuovo Testamento, mantenendo inalterata l'immagine evangelica di Maria.

\section{EWANGELICZNY OBRAZ MARYI MATKI JEZUSA W PIERWSZYCH TRZECH WIEKACH}

(Streszczenie)

Maryja, matka Jezusa z Nazaretu, jest czczona przede wszystkim jako Bogurodzica, czyli Theotókos, co jest terminem zaaprobowanym na Soborze Efeskim w 431 roku. Niektórzy teolodzy utrzymują, iż tytuł ten był używany w stosunku do matki Jezusa już znacznie wcześniej. W niniejszym artykule analizujemy, czy rzeczywiście byl on używany w pierwszych trzech wiekach; zatrzymujemy się tutaj nad sposobem w jakim Maryja jest przedstawiana przez autorów chrześcijańskich w tym okresie.

\footnotetext{
${ }^{59}$ Costantino avrebbe usato l'espressione $\Theta \varepsilon \circ \tilde{~} \mu$ intᄁ@, cf. D.F. Wright, Constantine and the „Mother of God": Oratio ad Sanctorum coetum 11:9, „Studia Patristica” 24 (1993) 355-359.

${ }^{60}$ Cf. R. Laurentin, Compendio, op. cit., p. 54, nota 2.

${ }^{61}$ Ibidem.
} 